\title{
How can we reduce the risks of SARS-CoV-2 (COVID-19) for dentists and their patients?
}

\section{Carlos Fernando de Almeida Barros Mourão, ${ }^{1,2}$ Kayvon Javid $^{2}$ and Igor Bastos Barbosa ${ }^{3}$}

\section{A commentary on}

\section{Yang Y, Soh H Y, Cai Z G, Peng X, Zhang Y, Guo C B.}

Experience of Diagnosing and Managing Patients in Oral Maxillofacial Surgery during the Prevention and Control Period of the New Coronavirus Pneumonia. Chin J Dent Res 2020; 23: 57-62.

\section{Abstract}

Design Special report.

Study population This paper presented a report about the experience of the oral and maxillofacial surgeons (OMS) of Peking University School and Hospital of Stomatology, during the COVID-19 (SARS-CoV-2) pandemic.

Data analysis The experience of that department formed the content of the report.

Results In this study, the authors presented an informative description of experience of treating patients under a pandemic condition. The authors offer some methods of trying to protect oro-maxillofacial surgeons, using an algorithm of diagnosis and classifying the risk of contamination and the materials required in order to avoid it.

Conclusions In conclusion, the authors suggest the use of the algorithm for patient admission during the COVID-19 outbreak.

\section{Commentary}

This study by Yang et al. offers some important points for patient management. ${ }^{1}$ It is not only relevant for reducing infection by COVID-19 in oral and maxillofacial surgeons but for all the dental community. Unfortunately, the present article only suggests personal protective measures for dental surgeons and omits consideration of patient health. The same problem exists in a recent report by Ather et al. ${ }^{2}$

Obviously, in the front line, treating the disease, there are many health professionals who are bravely fighting this invisible enemy, which can cause severe damage to humans and has led many patients and some health professionals to die.

With this pandemic, many governments have decided to keep the population in quarantine, trying to prevent the spread of the virus. As a result, many professionals, especially the self-employed, as in the case of most dentists, have had to close their clinics and dental offices. The return of dentists to clinical activities

\section{Practice points}

Three things should occur before any member of the dental team has contact with a patient. These are:

1. Patient history, preferably performed remotely, which can be done by phone or video call

2. One health professional or staff, under full PPE, to check the patient's temperature before entering the OMS department or dental office

3. A rapid test for COVID-19.

4. After these three steps, and if 3 is negative, the patient may be seen by the dental surgeon.

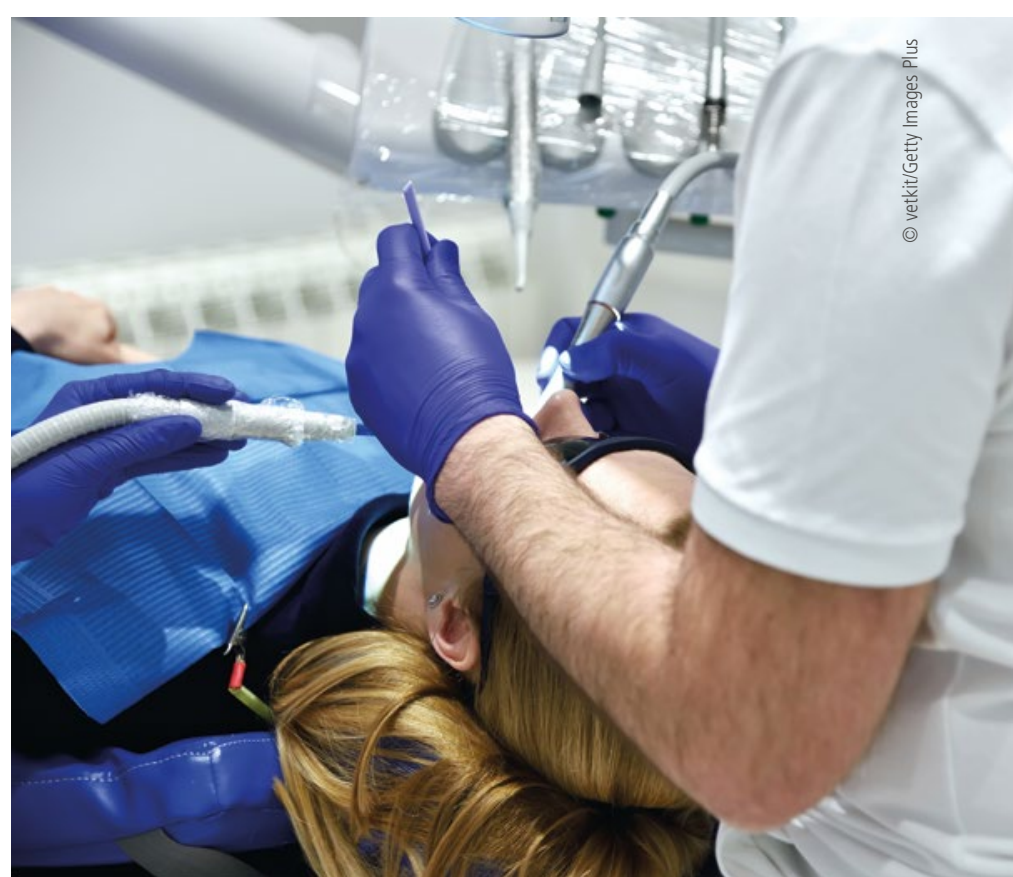

implies a range of changes in their office routine. This is necessary not only because governments require it but in order to avoid contamination by COVID-19 which is a highly transmissible virus. ${ }^{3,4}$ Care of both dental staff and patients must be rethought due to the pandemic.

This transmissibility is increased by the use of materials or methods that produce aerosols, such as those that happen in the hospitals and dental offices. Thus, the dental surgeon has become one of the professionals with the highest risk of contamination by COVID-19. ${ }^{5}$

Both articles suggest stricter protective measures during oral and maxillofacial surgery (OMS) or dental procedures, but nothing has actually been scientifically proven to prevent contamination of the dental surgeon by the virus. Equally, protective measures do not guarantee the non-contamination of other patients. It is speculated that COVID-19 can remain suspended in the environment for up to more than one hour. ${ }^{6}$ And with the production of aerosols, this 
could contaminate not only the specific area of care but with the air circulation, the entire environment of the hospital/dental office.

In addition to the use of individual protective equipment, such as masks, glasses and other protection, as described in Yang et al. ${ }^{1}$ and Ather et al., ${ }^{2}$ the authors suggest that three simple assessments should become routine. The first and most important is the patient's history, preferably performed remotely, which can be done by phone or video call. With this, it is possible to know if the patient has had contact with other infected people, or if they have symptoms that may suggest infection with COVID-19, or even if they already had the disease. Next, checking the patient's temperature before entering the dental office, and finally and most importantly is a rapid test in the dental office, which would indicate whether the patient is infected or not. If these tests are inconclusive or positive, care must be postponed, and the patient referred to the attending physician or healthcare providers for further testing, to ascertain whether the patient has COVID-19 and ensure that they are appropriately medically cared for and isolated.

With these three simple preventive measures, the dental surgeon and his team might be able to resume their activities with greater safety, reducing the risk of contamination for professionals and patients assisted.

\section{Author affiliations}

${ }^{1}$ Department of Oral Surgery, Dentistry School, Fluminense Federal University, Niterói, Rio de Janeiro - Brazil; ${ }^{2}$ South Bay Dental Institute, California - United States; ${ }^{3}$ Department of Endodontics, Dentistry School, Fluminense Federal University, Niterói, Rio de Janeiro, Brazil.

\section{References}

1. Yang Y, Soh H Y, Cai Z G, Peng X, Zhang Y, Guo C B. Experience of Diagnosing and Managing Patients in Oral Maxillofacial Surgery during the Prevention and Control Period of the New Coronavirus Pneumonia. Chin I Dent Res 2020; 23: 57-62.

2. Ather A, Patel B, Ruparel N B, Diogenes A, Hargreaves K M. Coronavirus Disease 19 (COVID-19): Implications for Clinical Dental Care. J Endod 2020; 46: 584595.

3. Woon Fong Leung W, Sun Q. Electrostatic Charged Nanofiber Filter for Filtering Airborne Novel Coronavirus (COVID-19) and Nano-aerosols. Sep Purif Technol 2020; 22: 116886 . DOI: $10.1016 /$ j.seppur.2020.116886.

4. Setti L, Passarini F, De Gennaro G et al. Airborne Transmission Route of COVID-19: Why 2 Meters/6 Feet of Inter-Personal Distance Could Not Be Enough. Int J Environ Res Public Health 2020; 23: 17. DOI 10.3390/ijerph17082932.

5. Polednik B. Aerosol and bioaerosol particles in a dental office. Environ Res 2014; 134: 405409.

6. van Doremalen $\mathrm{N}$, Bushmaker T, Morris D H et al. Aerosol and Surface Stability of SARS-CoV2 as Compared with SARS-CoV-1. N Engl / Med 2020; 382: 15641567.

Evidence-Based Dentistry (2020) 21, 50-51.

https://doi.org/ 10.1038/s41432-020-0092-8 